\title{
Foundation Flash 8
}

Sham Bhangal and Kristian Besley

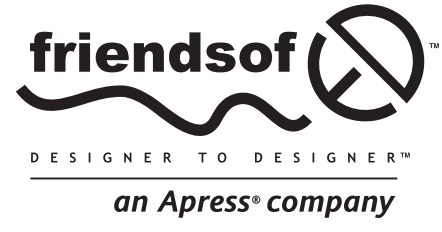




\title{
Foundation Flash 8
}

\author{
Copyright (c) 2006 by Sham Bhangal and Kristian Besley
}

All rights reserved. No part of this work may be reproduced or transmitted in any form or by any means, electronic or mechanical, including photocopying, recording, or by any information storage or retrieval system, without the prior written permission of the copyright owner and the publisher.

ISBN (pbk): 1-59059-542-4

Printed and bound in the United States of America 987654321

Distributed to the book trade worldwide by Springer-Verlag New York, Inc., 233 Spring Street, 6th Floor, New York, NY 10013.

Phone 1-800-SPRINGER, fax 201-348-4505, e-mail orders-ny@springer-sbm.com, or visit www.springeronline.com.

For information on translations, please contact Apress directly at 2560 Ninth Street, Suite 219, Berkeley, CA 94710. Phone 510-549-5930, fax 510-549-5939, e-mail info@apress.com, or visit www.apress.com.

The information in this book is distributed on an "as is" basis, without warranty. Although every precaution has been taken in the preparation of this work, neither the author(s) nor Apress shall have any liability to any person or entity with respect to any loss or damage caused or alleged to be caused directly or indirectly by the information contained in this work.

The source code for this book is freely available to readers at www. friendsofed. com in the Downloads section.

\author{
Credits \\ Lead Editor Assistant Production Director \\ Chris Mills Kari Brooks-Copony
}

Technical Reviewer

Todd Yard

Production Editor

Linda Marousek

Editorial Board

Steve Anglin, Dan Appleman, Ewan Buckingham, Gary Cornell,

Tony Davis, Jason Gilmore,

Jonathan Hassell, Chris Mills, Dominic Shakeshaft, Jim Sumser

Project Manager Beth Christmas

Copy Edit Manager

Nicole LeClerc

Copy Editors

Compositor and Artist

Katy Freer

Proofreader

Patrick Vincent

Indexer

Present Day Indexing

Cover Image Designer

Corné van Dooren

Interior and Cover Designer

Kurt Krames

Damon Larson and Liz Welch

\author{
Manufacturing Director \\ Tom Debolski
}

Case study illustrations courtesy of Gareth Southwell at www.woodpig.com 


\section{CONTENTS AT A GLANCE}

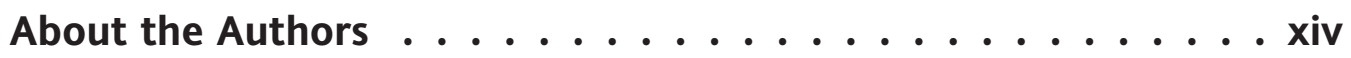

About the Technical Reviewer .............. . xv

About the Cover Image Designer $\ldots \ldots \ldots$ xvi

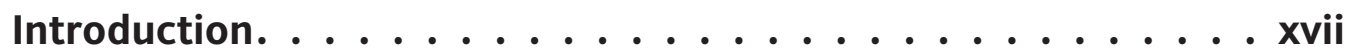

Chapter 1: Flash Movie Essentials . . . . . . . . . . 1

Chapter 2: The Flash Tools Panel . . . . . . . . . . . . 29

Chapter 3: Flash Symbols and Libraries . . . . . . . . . . 71

Chapter 4: Managing Content ............. 97

Chapter 5: Working with Color and Images . . . . . . . . 131

Chapter 6: Motion Tweening . . . . . . . . . . 175

Chapter 7 Shape Tweening . . . . . . . . . . 207

Chapter 8: Masks and Masking . . . . . . . . . . . 227

Chapter 9: Advanced Animation, Effects, and Commands. . . . . 249

Chapter 10: Actions and Interactions . . . . . . . . . . 301 
Chapter 11: Intelligent Actions . . . . . . . . . . . . 327

Chapter 12: Multimedia: Sound and Video . . . . . . . . . 353

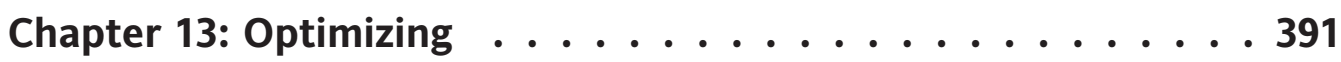

Chapter 14: Publishing . . . . . . . . . . . . . 415

Chapter 15: Intermediate ActionScript, Part $1 \ldots \ldots 37$

Chapter 16: Intermediate ActionScript, Part $2 \ldots . . . . . .473$

Chapter 17: High-Level Site Design . . . . . . . . . . . . 505

Chapter 18: Futurescape . . . . . . . . . . . . 519

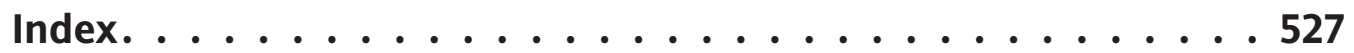




\section{CONTENTS}

About the Authors $\ldots \ldots \ldots$................ xiv

About the Technical Reviewer ............. xv

About the Cover Image Designer ............ xvi

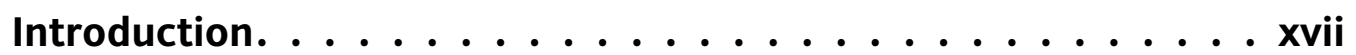

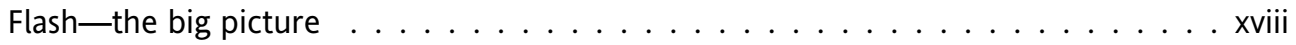

What's significant about Flash $8 \ldots \ldots \ldots \ldots \ldots \ldots$

Our aims and philosophy in this book . . . . . . . . . . . . . xxi

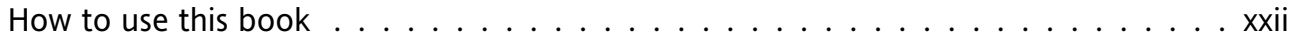

Chapter 1: Flash Movie Essentials . . . . . . . . . . . 1

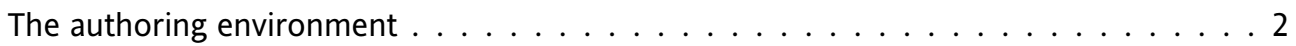

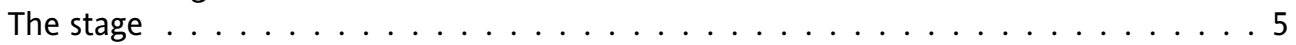

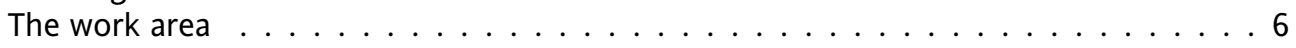

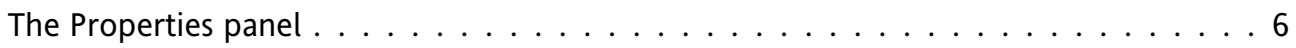

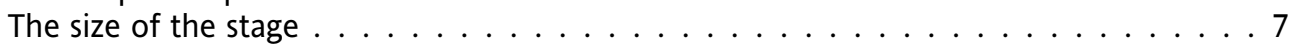

The movie's background color . . . . . . . . . . . . . . . . . . 9

Default movie settings . . . . . . . . . . . . . . . . . . 10

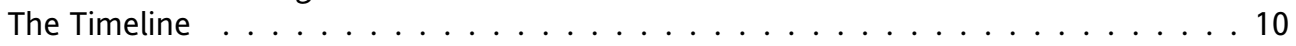

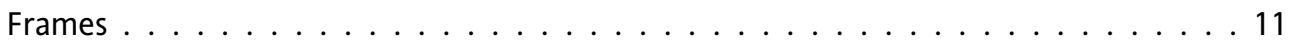

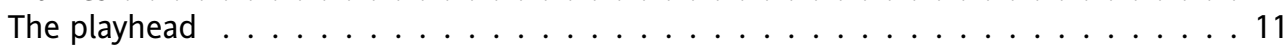

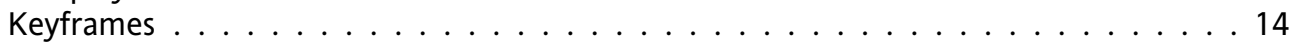

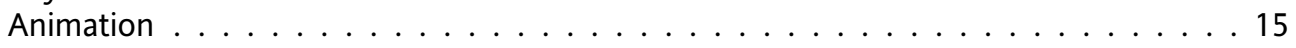

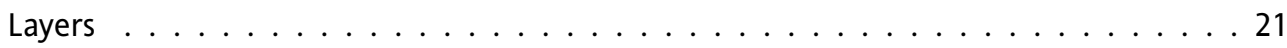

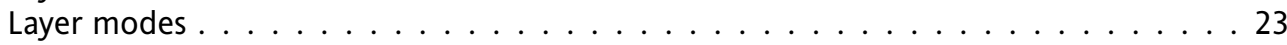

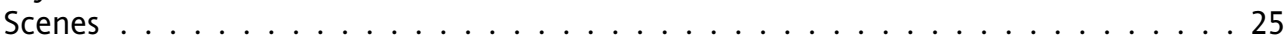

The Movie Explorer . . . . . . . . . . . . . . . . . . . . . . 26

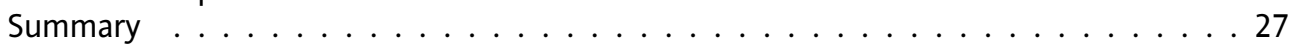


Chapter 2: The Flash Tools Panel . . . . . . . . . . . . . 29

The Tools panel . . . . . . . . . . . . . . . . . . . . . . 30

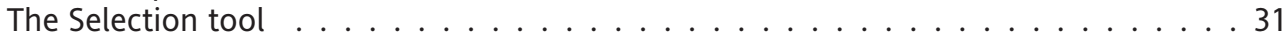

The Zoom tool . . . . . . . . . . . . . . . . . . . . . . . . 38

The Hand tool . . . . . . . . . . . . . . . . . . . . . . . . . . . . . . . . . 39

The Tools panel's drawing and modification tools . . . . . . . . . . . . . . . . . 39

The Rectangle tool . . . . . . . . . . . . . . . . . . . . . 39

The Oval tool . . . . . . . . . . . . . . . . . . . . . . 41

The Line tool . . . . . . . . . . . . . . . . . . . . . . 43

The Paint Bucket tool . . . . . . . . . . . . . . . . . . . . 48

The Ink Bottle tool . . . . . . . . . . . . . . . . . . . . . . . 49

The Eyedropper tool . . . . . . . . . . . . . . . . . . . . . 49

The Polystar tool . . . . . . . . . . . . . . . . . . . . . . 50

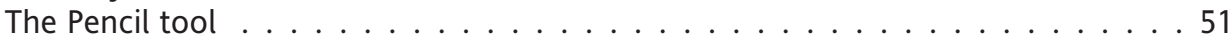

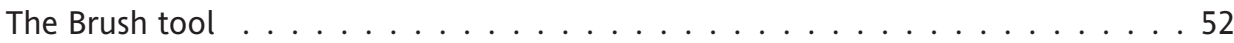

The Eraser tool . . . . . . . . . . . . . . . . . . . . . . 55

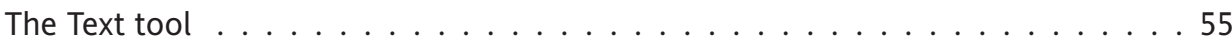

The Free Transform tool . . . . . . . . . . . . . . . . . . . . . . 58

The Pen tool . . . . . . . . . . . . . . . . . . . . . . 63

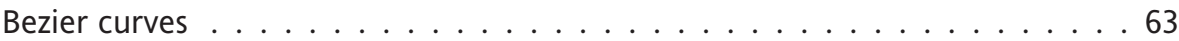

The Subselect tool . . . . . . . . . . . . . . . . . . . . . . . 664

Beziers and animation . . . . . . . . . . . . . . . . 665

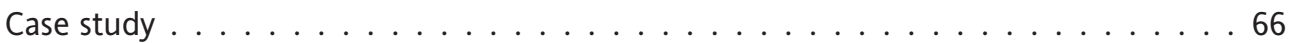

Summary . . . . . . . . . . . . . . . . . . . . . . 69

Chapter 3: Flash Symbols and Libraries . . . . . . . . . . 71

Symbol essentials . . . . . . . . . . . . . . . . . . . . . . . 72

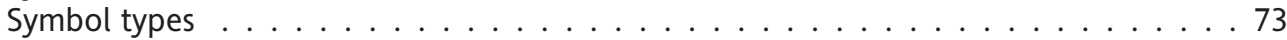

Graphic symbols . . . . . . . . . . . . . . . . . . . . . 73

Modifying symbols . . . . . . . . . . . . . . . . . . . . . . 79

Working with multiple Libraries . . . . . . . . . . . . . . . . 81

Button symbols . . . . . . . . . . . . . . . . . . . . 83

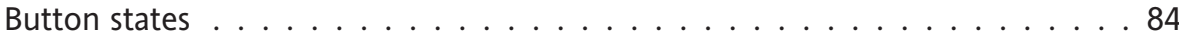

Movie clip symbols . . . . . . . . . . . . . . . . . . . . . . . . . . 878

Movie clips and the main Timeline . . . . . . . . . . . . . . . . 89

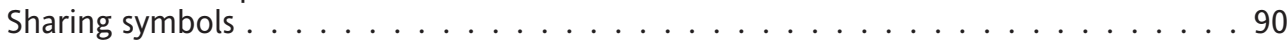

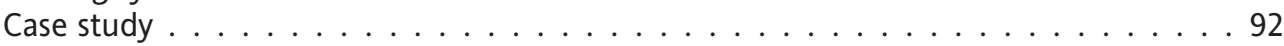

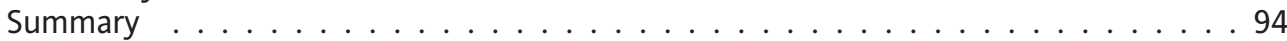

Chapter 4: Managing Content . . . . . . . . . . . 97

Grouped objects . . . . . . . . . . . . . . . . . . . . . . 98

Symbols within symbols . . . . . . . . . . . . . . . . . . 100

Snap Align . . . . . . . . . . . . . . . . . . . . . . . 105

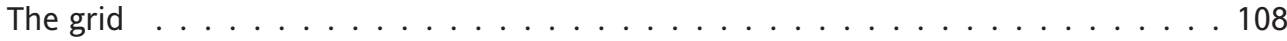

Rulers and guides . . . . . . . . . . . . . . . . . . . . 110

Alignment . . . . . . . . . . . . . . . . . . . . . 112

Align panel . . . . . . . . . . . . . . . . . . . . . 112 
Stacking order . . . . . . . . . . . . . . . . . . . . . . 117

Distribute to Layers option . . . . . . . . . . . . . . . . . . . . . 121

Layer folders . . . . . . . . . . . . . . . . . . . . . 122

History panel . . . . . . . . . . . . . . . . . . . . . . 123

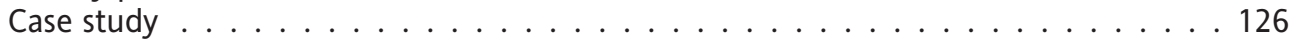

Summary . . . . . . . . . . . . . . . . . . . . . . . . . 129

Chapter 5: Working with Color and Images . . . . . . . . 131

Color primer . . . . . . . . . . . . . . . . . . . . . . . . . 132

Custom colors . . . . . . . . . . . . . . . . . . . . . . . 133

Persistent custom colors . . . . . . . . . . . . . . . . . . . . . . . . 138

Gradient color . . . . . . . . . . . . . . . . . . . . . . . . . . . . . . . 140

Making the gradient . . . . . . . . . . . . . . . . . . . . . 141

Using light and shade with gradients . . . . . . . . . . . . . . . . . . . . . . . 144

Applying gradients to objects and modifying them . . . . . . . . . . . . . . . 146

Overflowing fills . . . . . . . . . . . . . . . . . . . . . . . 151

Locking fills . . . . . . . . . . . . . . . . . . . . . . 153

Bitmaps . . . . . . . . . . . . . . . . . . . . . . 154

Using bitmap images in Flash . . . . . . . . . . . . . . . . . . 154

Tracing bitmaps . . . . . . . . . . . . . . . . . . . . . 158

Optimizing bitmaps . . . . . . . . . . . . . . . . . . . 159

GIF files . . . . . . . . . . . . . . . . . . . . . . . . . . . 160

Understanding transparent GIFs . . . . . . . . . . . . . . . . . . 160

Understanding animated GIFs . . . . . . . . . . . . . . . . . . . . . 161

JPEG files . . . . . . . . . . . . . . . . . . . . . . . 162

Fireworks files . . . . . . . . . . . . . . . . . . . . . . 164

Importing vector images into Flash . . . . . . . . . . . . . . 165

Importing FreeHand files into Flash . . . . . . . . . . . . . . 165

Importing PDF documents into Flash . . . . . . . . . . . . . . 166

Vector clip art . . . . . . . . . . . . . . . . . . . . . . 168

Fonts and typefaces . . . . . . . . . . . . . . . . . . . . 169

Working with device fonts . . . . . . . . . . . . . . . . 169

Working with text . . . . . . . . . . . . . . . . . . 170

Case study . . . . . . . . . . . . . . . . . . . . . 170

Summary . . . . . . . . . . . . . . . . . . . . . . 173

Chapter 6: Motion Tweening . . . . . . . . . . . 175

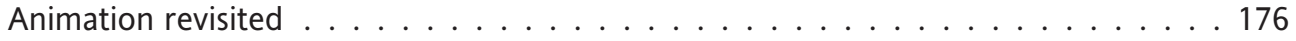

Motion tweens . . . . . . . . . . . . . . . . . . . . . . . . 177

Scaling objects in motion tweens . . . . . . . . . . . . . . . . . 179

Easing . . . . . . . . . . . . . . . . . . . . . 181

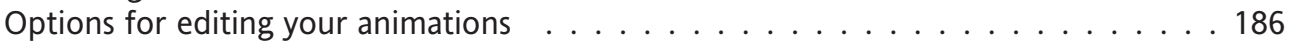

Using onion skins . . . . . . . . . . . . . . . . . 186

Editing multiple frames . . . . . . . . . . . . . . . . . . . . . . 188

Motion tween effects . . . . . . . . . . . . . . . . . . . . . 189

Color change animations . . . . . . . . . . . . . . . . . . . . . . . 191

Motion guides . . . . . . . . . . . . . . . . . . . . . 193

Case study . . . . . . . . . . . . . . . . . . . . . 200

Summary . . . . . . . . . . . . . . . . . . . . 205 
Chapter 7: Shape Tweening . . . . . . . . . . . . 207

Shape tweens . . . . . . . . . . . . . . . . . . . . . 208

How shape tweens work . . . . . . . . . . . . . . . . . . . . . 208

Irregular shapes . . . . . . . . . . . . . . . . . . . . . 210

Common tweening text effects . . . . . . . . . . . . . . . . . 212

Natural-looking tweens . . . . . . . . . . . . . . . . . 215

Tweens with color . . . . . . . . . . . . . . . . . . . . . . 216

Shape tween modifiers . . . . . . . . . . . . . . . . . 217

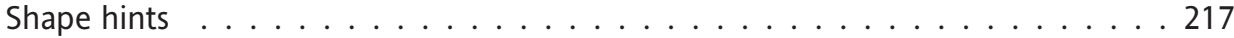

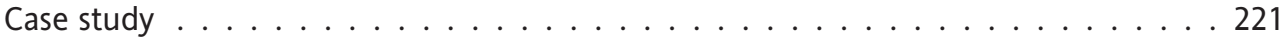

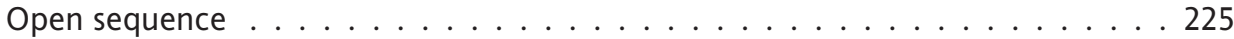

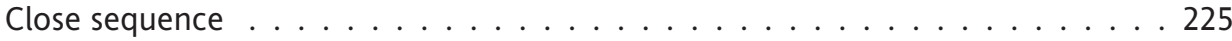

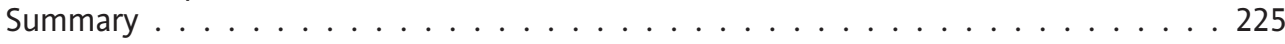

Chapter 8: Masks and Masking . . . . . . . . . . 225

What is a mask ? . . . . . . . . . . . . . . . . . . . . . . . . . 228

Animated masks and masking multiple layers . . . . . . . . . . . . . . 235

Using masks with text . . . . . . . . . . . . . . . . . . . . . 237

A text-shaped mask . . . . . . . . . . . . . . . . . . . . . 238

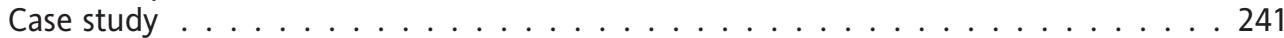

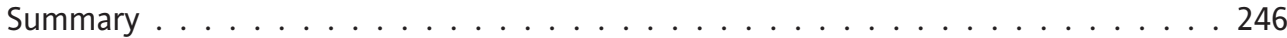

Chapter 9: Advanced Animation, Effects, and Commands. . . . . 249

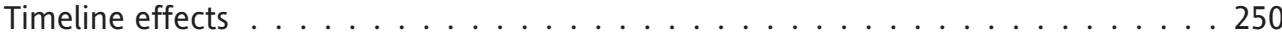

Other kinds of timeline effects . . . . . . . . . . . . . . . . . . . . 256

Assistants. . . . . . . . . . . . . . . . . . 256

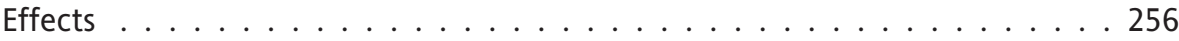

Transform/transition . . . . . . . . . . . . . . . . 256

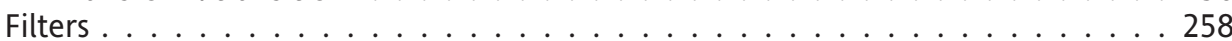

Blend modes . . . . . . . . . . . . . . . . . . . . . . . . . . 268

Nested blend modes . . . . . . . . . . . . . . . . . . . . . . . . . 271

Layer blending . . . . . . . . . . . . . . . . . . . . . . . . 271

Alpha blending . . . . . . . . . . . . . . . . . . . . . . 272

Erase blends . . . . . . . . . . . . . . . . . . . . . . . . . 273

Automated commands . . . . . . . . . . . . . . . . . . . . . . . 274

Reacquainting yourself with the History panel . . . . . . . . . . . . . . . . . . 274

Creating a reusable command . . . . . . . . . . . . . . . . . . . . . . . . 277

Behaviors . . . . . . . . . . . . . . . . . . 278

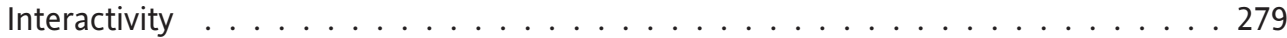

Events and event handlers . . . . . . . . . . . . . . . . . . . 279

Buttons as interactive elements . . . . . . . . . . . . . . . . . . . 280

Symbols on their own do not create interactivity . . . . . . . . . . . . . . 280

Movie clips and buttons only behave on a timeline . . . . . . . . . . . . . . 281

Creating animated buttons . . . . . . . . . . . . . . . . . . . . . . 284

Creating buttons that talk . . . . . . . . . . . . . . . . 285

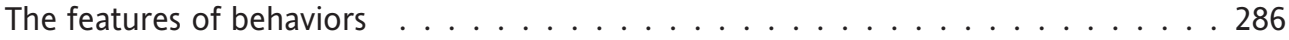

Attaching behaviors to frames and buttons . . . . . . . . . . . . . 289

Case study . . . . . . . . . . . . . . . . . . . . . . . 292

Summary . . . . . . . . . . . . . . . . . . . . . . 298 
Chapter 10: Actions and Interactions . . . . . . . . . . . 301

Actionscript vs. behaviors . . . . . . . . . . . . . . . . . . . . . 302

Scripts, programs, and code-it's all Geek/Greek to me . . . . . . . . . . . . . . 303

Programming and scripting . . . . . . . . . . . . . . . 303

Scripting and animation . . . . . . . . . . . . . . . . . . . 304

Understanding scripting . . . . . . . . . . . . . . . . . . . . 304

Syntax . . . . . . . . . . . . . . . . . . . . . 304

Rule 1: End each line with a semicolon . . . . . . . . . . . . . . . . . . 305

Rule 2: The object, method, and argument . . . . . . . . . . . . 305

Rule 3: The code block . . . . . . . . . . . . . . . . . . . . 306

Camel case . . . . . . . . . . . . . . . . . . . . . 307

Indenting . . . . . . . . . . . . . . . . . . . 307

Use code blocks often . . . . . . . . . . . . . . . . . . . . . 307

The Flash scripting environment . . . . . . . . . . . . . . . . . . 308

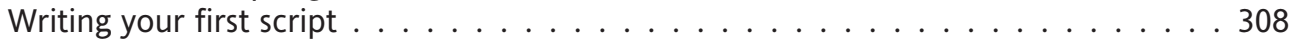

Linking your movie to a URL . . . . . . . . . . . . . . . . . . . . . 313

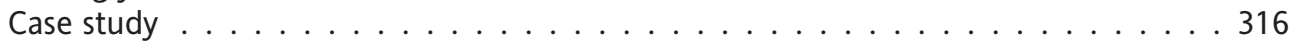

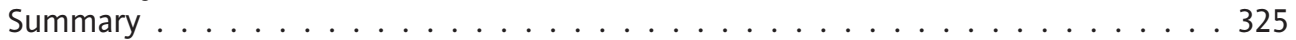

Chapter 11: Intelligent Actions . . . . . . . . . . . . 327

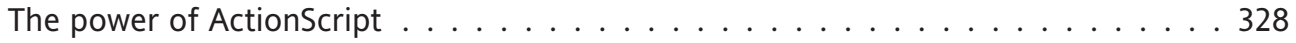

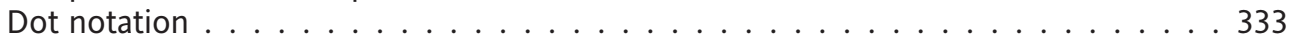

Teaching your movie to think for itself . . . . . . . . . . . . . . . . 341

Variables . . . . . . . . . . . . . . . . . . . . . . . . 342

Conditional statements . . . . . . . . . . . . . . . . . . . . . . . 344

Case study . . . . . . . . . . . . . . . . . . . . . . . . . 347

Summary . . . . . . . . . . . . . . . . . . . . . . . . . 351

Chapter 12: Multimedia: Sound and Video . . . . . . . . . . 353

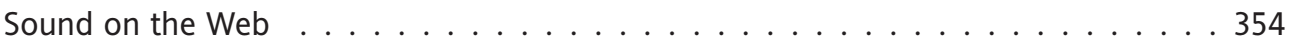

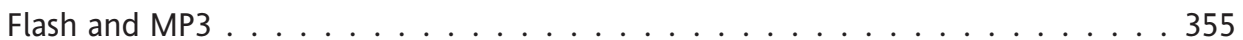

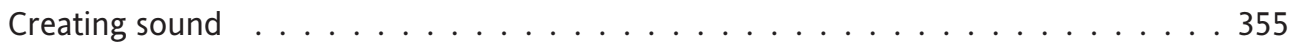

Importing and exporting sound with Flash . . . . . . . . . . . 356

Using sound in Flash . . . . . . . . . . . . . . . . . . . . . . 360

Attaching sounds to the Timeline . . . . . . . . . . . . . . . 360

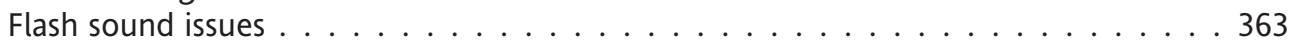

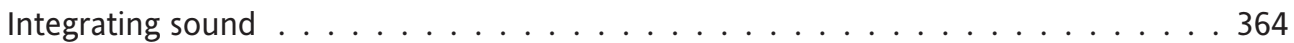

Video on the Web . . . . . . . . . . . . . . . . . . . . . . . 370

Creating video . . . . . . . . . . . . . . . . . . . . . . . 371

Flash-friendly formats . . . . . . . . . . . . . . . . . . . . . 372

Using video with Flash . . . . . . . . . . . . . . . . . . . . . . . 372

Editing video clips in Flash . . . . . . . . . . . . . . . . . . . . . . 377

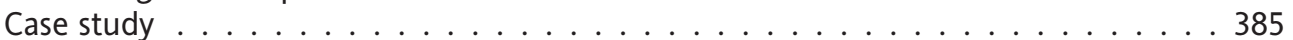

Importing and placing the videos . . . . . . . . . . . . . . . . 386

Summary . . . . . . . . . . . . . . . . . . . . . . . . . . 388 
Chapter 13: Optimizing . . . . . . . . . . . . 391

Flash and the Internet . . . . . . . . . . . . . . . . . . . 392

The Internet . . . . . . . . . . . . . . . . . . . . . . . . 392

The World Wide Web . . . . . . . . . . . . . . . . . . . . . . 393

Bandwidth . . . . . . . . . . . . . . . . . . 393

The end user-your audience . . . . . . . . . . . . . . . . . . . . . 394

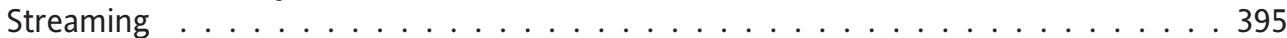

The Bandwidth Profiler . . . . . . . . . . . . . . . . . . . . . 396

Optimizing and fine-tuning Flash movies . . . . . . . . . . . . . . . . . . . . . 401

Structure . . . . . . . . . . . . . . . . . . . . . 402

Use the right components in your movie . . . . . . . . . . . . . . . . . . . . 402

Optimizing elements . . . . . . . . . . . . . . . . . . . . . 403

Optimizing tricks and tips . . . . . . . . . . . . . . . . . . . . . 404

Loading multimedia on demand . . . . . . . . . . . . . . . . . . . . 405

Loading video files, MP3s, and JPEGs . . . . . . . . . . . . . . . . . . . 407

Video . . . . . . . . . . . . . . . . . . . . . . 407

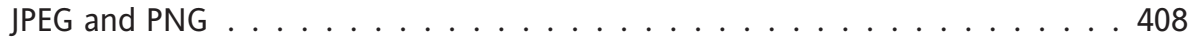

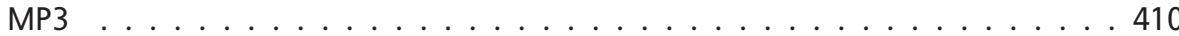

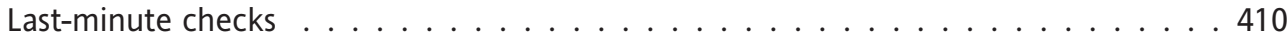

Case study . . . . . . . . . . . . . . . . . . . . . . . 410

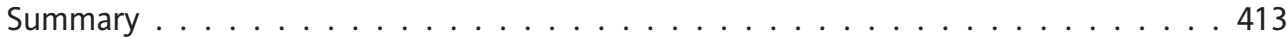

Chapter 14: Publishing . . . . . . . . . . . . . 415

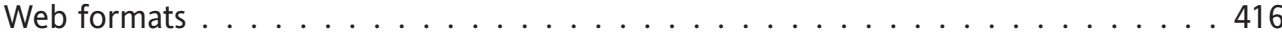

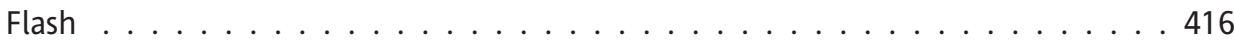

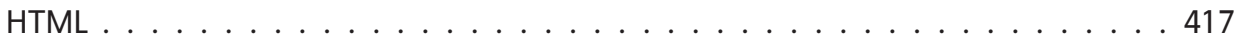

Animated GIFs . . . . . . . . . . . . . . . . . . . . 418

QuickTime . . . . . . . . . . . . . . . . . . . . . 418

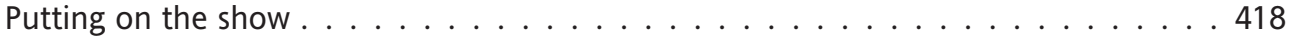

Flash file publishing options . . . . . . . . . . . . . . . . 425

HTML file publishing options . . . . . . . . . . . . . . . . 426

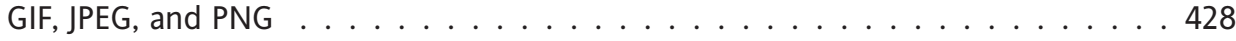

QuickTime movies . . . . . . . . . . . . . . . . . . 428

Projector files . . . . . . . . . . . . . . . . . . . . . . . . 429

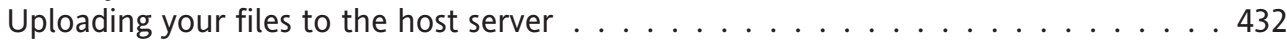

Case study . . . . . . . . . . . . . . . . . . . . . 433

Summary . . . . . . . . . . . . . . . . . . . . . . . 434

Chapter 15: Intermediate ActionScript, Part $1 \ldots$. . . . . . 437

Planning your complex ActionScript: A practical overview . . . . . . . . . . . . . 438

Defining a problem . . . . . . . . . . . . . . . . . . . . 438

Breaking down the Smiler problem . . . . . . . . . . . . . . . . . . . 439

Basic input and output . . . . . . . . . . . . . . . . . . . . . . . 441

Referencing paths with dot notation . . . . . . . . . . . . . . . . . . 445

Many movie clips, many variables . . . . . . . . . . . . . . . . . . . . . . 448 
Dynamic animation . . . . . . . . . . . . . . . . . . . . . . . . 454

Creating advanced animation communication schemes . . . . . . . . . . . . . . . 461

Making Smiler age-sensitive . . . . . . . . . . . . . . . . . . . . . . . . . 464

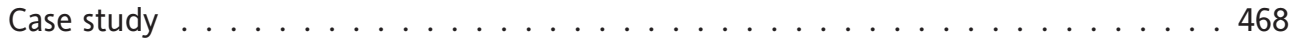

Summary . . . . . . . . . . . . . . . . . . . . . . . . 470

Chapter 16: Intermediate ActionScript, Part $2 \ldots . . . . . .473$

Making coffee . . . . . . . . . . . . . . . . . . . . . . 474

To make a cup of Café au Lait (coffee with milk) and sugar . . . . . . . . . . . . . 474

A bottom-up cup of coffee . . . . . . . . . . . . . . . . . . . . . . 474

Creating the super class . . . . . . . . . . . . . . . . . . . . . . 477

Reviewing the solution . . . . . . . . . . . . . . . . . . . . . . . . . . . . . . . . . . . . . .

Flash and the built-in classes . . . . . . . . . . . . . . . . . . . . . . . 482

A simple mouse trail . . . . . . . . . . . . . . . . . . . . . . . . 483

Putting it all together . . . . . . . . . . . . . . . . . . . . . . 488

Possible improvements and modifications to your game . . . . . . . . . . 502

Summary . . . . . . . . . . . . . . . . . . . . . . 503

Chapter 17: High-Level Site Design . . . . . . . . . . 505

The principles of good design . . . . . . . . . . . . . . . . . . 506

File structure and file size . . . . . . . . . . . . . . . . . . . . 506

Preloaders . . . . . . . . . . . . . . . . . . . . . . . 507

Basic: Timeline-based loader . . . . . . . . . . . . . . . . . . . . . . . 507

Intermediate: ActionScript-based loader . . . . . . . . . . . . . . . . 507

Intros . . . . . . . . . . . . . . . . . . . . . . . . 508

Tailoring your designs . . . . . . . . . . . . . . . . . . . . . 508

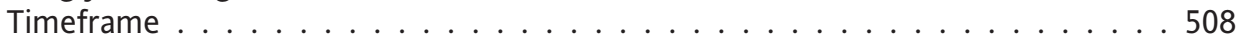

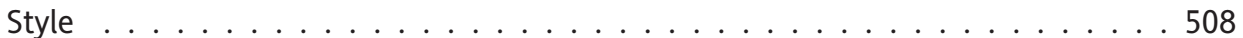

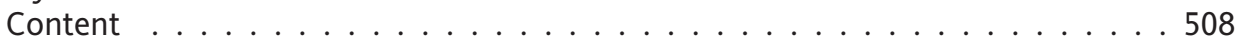

Navigation . . . . . . . . . . . . . . . . . . . . . . 508

Case study 1 : online showcase . . . . . . . . . . . . . . . . . . . 509

Storyboarding . . . . . . . . . . . . . . . . . . . . 509

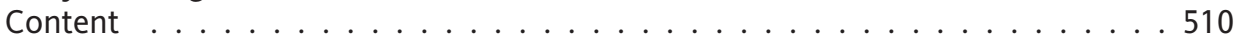

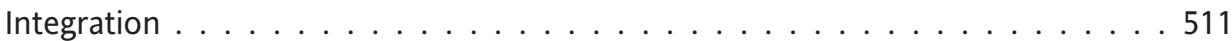

Summary . . . . . . . . . . . . . . . . . . . . . . . . . 512

Case study 2: my Flash home site interface . . . . . . . . . . . . . . . . . 512

Random idea \#1 . . . . . . . . . . . . . . . . . . . . . . 513

Random idea \#2 . . . . . . . . . . . . . . . . . . . . . . 513

Integration . . . . . . . . . . . . . . . . . . . . . . . 514

Summary . . . . . . . . . . . . . . . . . . . . . . . . . 514

Dynamic websites . . . . . . . . . . . . . . . . . . 515

Case study 3 : a dynamic visual guestbook . . . . . . . . . . . . . . . 515

Suggested sites . . . . . . . . . . . . . . . . . . . . . 516

Summary . . . . . . . . . . . . . . . . . . . . . . . . . . . 517 
Chapter 18: Futurescape . . . . . . . . . . . . 519

Where next? . . . . . . . . . . . . . . . . . . . . . 520

Using and improving your Flash skills . . . . . . . . . . . . . . . . . . 520

Flash site design . . . . . . . . . . . . . . . . . . . . . . . . . . . 521

Flash games and toys . . . . . . . . . . . . . . . . . . . . . . 521

Flash cartoons . . . . . . . . . . . . . . . . . . . . 521

Using Flash with other software . . . . . . . . . . . . . . . . . . 521

3D in Flash . . . . . . . . . . . . . . . . . . . . . . . . 522

Advanced ActionScript-based Flash interface designs . . . . . . . . . . . . . . . 522

Dynamic content . . . . . . . . . . . . . . . . . . . . . 522

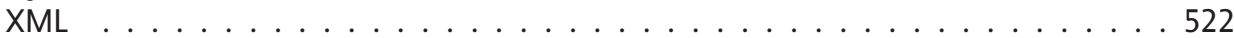

Starting a career in Flash . . . . . . . . . . . . . . . . . . . . 523

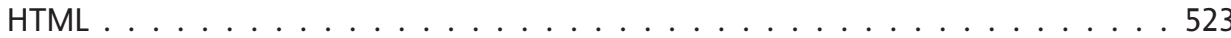

Graphic design . . . . . . . . . . . . . . . . . . . . . 523

Getting in . . . . . . . . . . . . . . . . . . . . 523

Flash farewell-for now . . . . . . . . . . . . . . . . . . . . 524

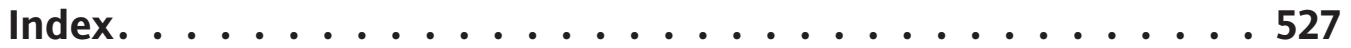




\section{ABOUT THE AUTHORS}

Sham Bhangal began on the route to web design in 1991, designing and specifying information screens for safety critical computer systems, as used in places like nuclear power plant control rooms. He soon discovered that more conventional interface design, animation, and multimedia tools were available, such as SoftimageXSI, Photoshop, and Flash. He has been writing books and articles on them since the turn of the century.

As well as being an author by night, Sham works as a Flash developer for an e-learning company during the day, creating online testing applications, simulations, and other web-based teaching applications using lots of class-based Actionscript and a fair bit of user interface and content design. He is also known to engage in copious amounts of freelance web design work.

Like all professional web designers, he never sleeps because new ideas and old clients have a habit of keeping him awake. You can catch up with him at boy@futuremedia.org.uk, or through his weblog, http://weblog.motion-graphics.org/.

Kristian Besley is a Flash/web developer currently working in an educational establishment and specializing in interactivity and dynamic-driven content (using ASP and PHP). He is also a lecturer in multimedia.

He has written a number of books, including Flash MX Video, Learn Programming with Flash $M X$, and the Foundation Flash series. He was a contributor to Flash Math Creativity, Flash MX Games Most Wanted, and Flash Video Creativity. He is also a contributor to Computer Arts magazine and has produced freelance work for numerous clients, including the BBC.

Kristian was born and currently resides in Swansea, Wales. He is a fluent Welsh speaker and is the creator of the first ever Welsh translation search plug-in for Firefox and Mozilla (available from http://mycroft.mozdev.org).
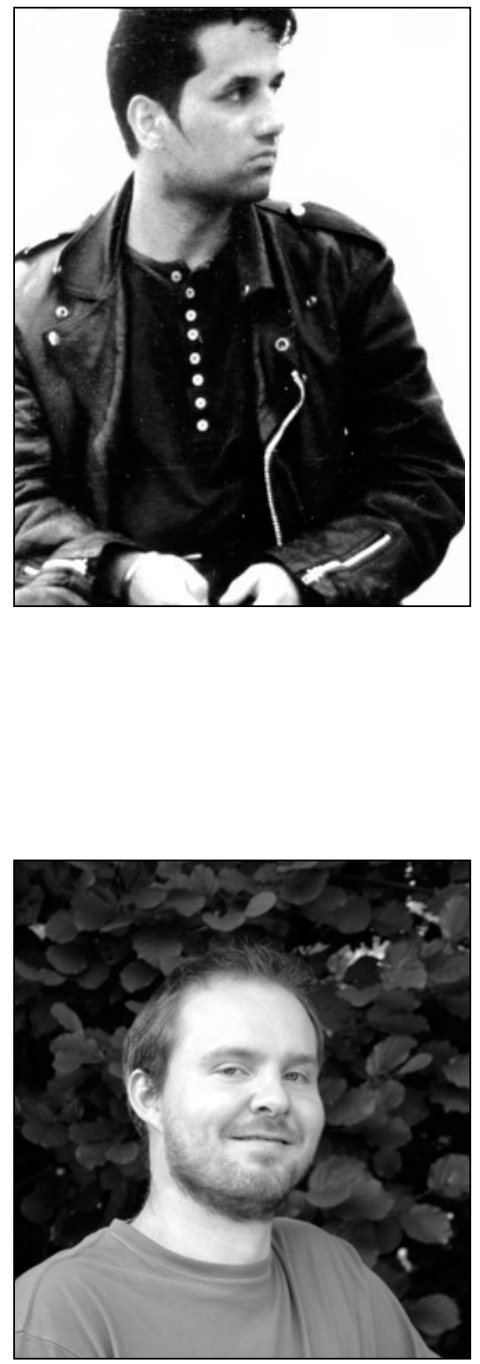

Portrait image courtesy of Simon James at www. thefresh.co.uk. 


\section{ABOUT THE TECHNICAL REVIEWER}

Todd Yard is a lead Flash developer at Brightcove (www.brightcove.com) in Cambridge, Massachusetts, and has contributed as an author or technical editor on 11 other friends of ED Flash books, including Flash 8 Essentials and Extending Flash MX 2004. 


\section{ABOUT THE COVER IMAGE DESIGNER}

Corné van Dooren designed the front cover image for this book. Having been given a brief by friends of ED to create a new design for the Foundation series, he was inspired to create this new setup combining technology and organic forms.

With a colorful background as an avid cartoonist, Corné discovered the infinite world of multimedia at the age of 17-a journey of discovery that hasn't stopped since. His mantra has always been "The only limit to multimedia is the imagination," a mantra that is keeping him moving forward constantly.

After enjoying success after success over the past yearsworking for many international clients, as well as being featured in multimedia magazines, testing software, and working on many other friends of ED books-Corné decided it was time to take another step in his career by launching his own company, Project 79, in March 2005.

You can see more of his work and contact him through www. cornevandooren. com or wWw. project79.com.

If you like his work, be sure to check out his chapter in New Masters of Photoshop: Volume 2, also by friends of ED (ISBN: 1-59059-315-4).

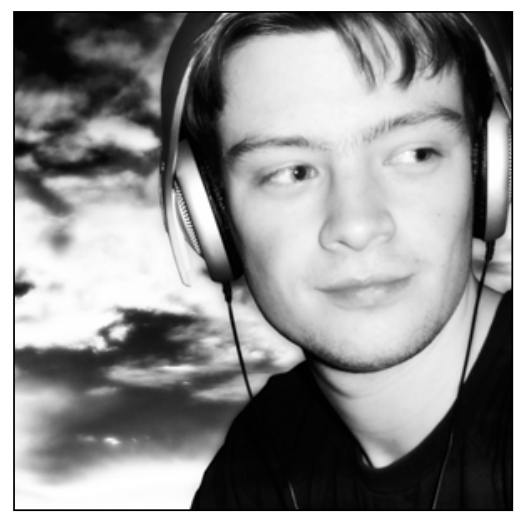

.

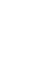

(

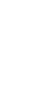




\section{INTRODUCTION}

This book aims to give you a solid foundation in the most essential skills you need to use Flash 8both the Basic and Professional versions. By the end of the book, you'll understand how the components of a Flash movie fit together, you'll have used all of the key tools, and you'll have integrated all your learning in a series of detailed creative exercises. Our mission is to launch you into orbit around planet Flash, equipped with all the tools and knowledge you need to make a safe landing.

Flash is one of the hottest content-creation technologies on the Web. From its origins as an animation package, Flash has grown stronger and planted deep roots. It is already used to create all kinds of content, such as website front-ends, interactive games, animated cartoons, movie trailers, and PDA interfaces. Perhaps its most significant role, however, is in creating interfaces for all these different areas. Its ability to present a clean, friendly, and functional front-end to the user is coupled with its power behind the scenes. Designers love Flash for its speed, quality, ease of use, and clearly structured functionality, and at the same time, both programmers and designers can use its Actionscript programming language to produce phenomenal results. Whatever kind of interface you want to build, Flash has the answer. If you've never used Flash before, you're in for a real treat.

As the Internet has changed, Flash has moved with it, evolving into a two-tier system. The timelinebased animation is still there, but it is underpinned by a stronger emphasis on functionality that enables you to create the large, code-heavy sites required for today's e-commerce front-ends, e-learning applications, and other intelligent user interfaces. Don't worry if you are not a heavy-duty programmer, though; you can still build Flash sites using many of the new features aimed directly at designers who are not programmers:

- If you are experienced in bitmap editing applications such as Adobe Photoshop, you will be able to leverage this experience in Flash, because Flash now supports many of the features seen in Photoshop, such as filters and blend modes.

- If you are more of an Illustrator/Freehand person, then you're also going to like Flash, because its new Object Drawing mode will make you feel right at home.

- If you are a video content producer, then Flash is also for you, because it is now one of the most powerful over-the-Web video-delivery systems.

- If you are a musician, then be prepared to learn a system that allows you to give your creations the web presence they deserve: 32-channel sound at the quality and format of your choosing! 
- Finally, if you are a traditional web designer, then you are in for a treat, because Flash already supports many of the things you are already familiar with (ActionScript, a JavaScript-like scripting language; support for subsets of both CSS and HTML; and runtime import of most of the common bitmap formats you are already used to). It also seamlessly addresses some of the things you have always had trouble with, such as consistent color, one single plug-in for all multimedia types (sound, video, animation), embedded fonts, multilanguage localization, and of course, a good interactive web animation system. Flash also addresses areas in which you may have previously thought Flash was weak, such as accessibility and security.

This book will take you step by step through every aspect of designing your own Flash interface, building your knowledge and skills with each chapter. We'll also look at the pitfalls and practicalities that every Flash web designer faces, teach you how to make your designs web friendly, and ensure that you know how to get your hard work up on the Net. But before we dive into these complex issues, let's make sure you know the basics of how Flash works, and why it's such a capable authoring tool.

\section{Flash-the big picture}

When you create a Flash movie for the Web, you're pulling together images, sound, video, text, and animation, and bundling them in a file that gets posted up on a website.

The Flash software you install on your computer is the authoring environment in which you create your masterpiece. The work in progress is stored in a file with the extension . fla. Once you're happy with your movie and you want to publish it to the Internet, Flash will convert the FLA file into a playable file with the extension . swf-pronounced "swiff" in the Flash community. The SWF file is then linked to an HTML file on the server that hosts your site:

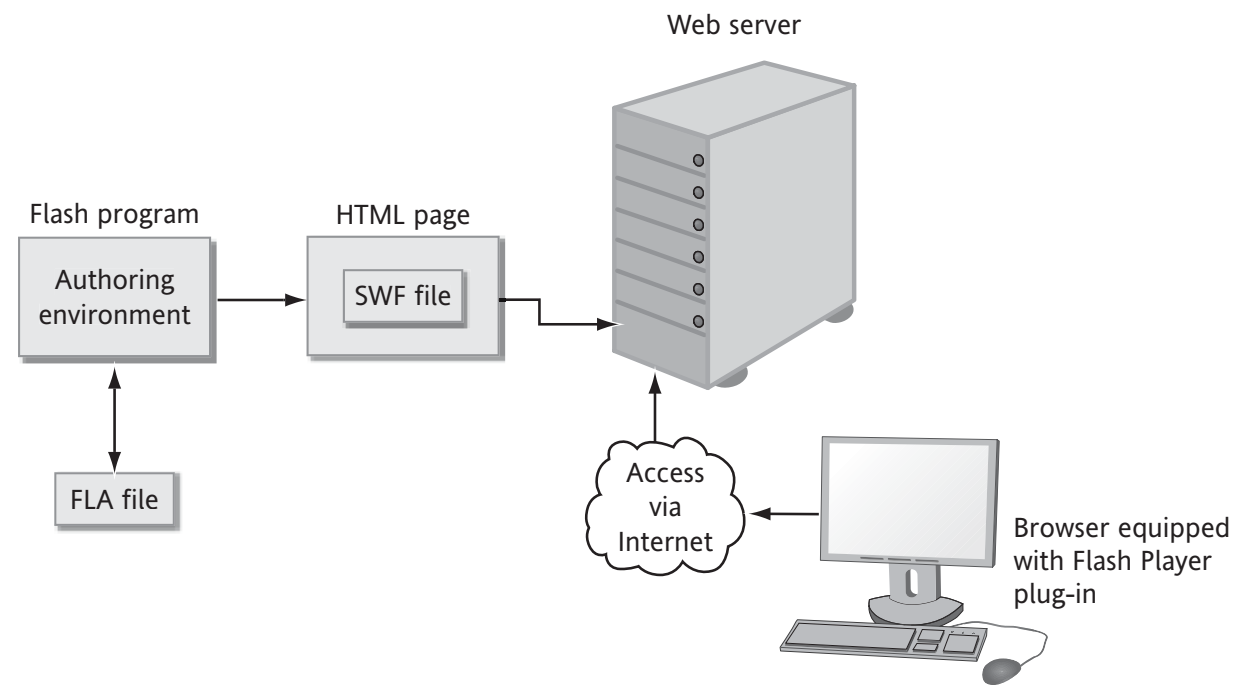

When a user visits your site, the SWF file is downloaded into their browser and your movie is played back. All the viewer needs is the Flash Player installed on his machine. This player is a reasonably compact download, and the vast majority of the world's browsers are equipped to play back Flash content.

\section{Xviii}


One of the reasons that Flash is such a popular tool is that it uses vector graphics technology. There are two main graphic standards on the Internet: raster (bitmaps) and vector. The majority of static images that you see on the Web are raster images, composed of files in formats such as BMP, GIF, and JPG. Raster images do a good job, but a big raster image usually requires a large file size, and a large file size means a long download time. And on the Web, download time is everything. Internet users are picky —if a site's packed with raster images and is taking too long to load, they'll just skip it and go somewhere else. This is where vectors come in. They're small, fast, and funky.

\section{Vector images describe the image in terms of coordinates and mathematical transforma- tions. That sounds complicated, but it's really as simple as saying, "Put a dot here, put a dot there, and draw a line between them." This compares with the raster technique of describing the color and position of every single pixel in the image.}

Vector graphic files are much more compact and efficient compared to rasters, and Flash is the main tool for delivering vector graphics and vector-based animations on the Web. The files that Flash creates are therefore comparatively small, which is one of the reasons for Flash's success.

A well-constructed Flash file will also stream onto the user's computer. That means it will load the first part of the animation and start playing it back while the rest of the animation loads in the background. Streaming a file correctly is an important technique for a Flash designer because it means that visitors are presented with something visual and enticing almost immediately-removing the danger that they will get bored and go elsewhere instead of waiting for the site to download.

Another disadvantage of raster images is that they're display dependent, meaning that if you create them to look just right on one particular display, the image could come out significantly altered if someone uses a different display resolution to view it. In addition, if you zoom in on a raster image, the pixels just get bigger and bigger until you end up with a screen full of squares of color that are completely unrecognizable as the source image. Vector images, though, can work independently of the display because the line will always be the same relative length and clarity no matter what resolution you use to view it. Also, no matter how far you zoom into a vector, the image will still stay crisp and at full resolution.

Why would you ever want to use a raster? Raster formats are good for images with thousands of different colors. Can you imagine trying to describe a photograph in terms of vectors? It would be horribly complicated, and you'd wind up with a far bigger file size compared to the raster equivalent. Luckily, Flash has the best of both worlds: the vast majority of its drawings and animations are vector-based, but when you need the extra richness that you can only get with a raster, Flash will allow you to import a bitmap and use it in conjunction with the dominant vectors.

Despite the fact that Flash is normally associated with vectors, Flash 8 now also includes many features normally associated with bitmaps, and this feature makes Flash much more accessible to people who are coming to Flash from more traditional digital art disciplines (especially graphic design and traditional web design). 


\section{What's significant about Flash 8}

If you've used a previous version of Flash, the first thing you'll notice is. . .er. . . that it appears like nothing much has changed! Looks can be deceptive, though, because a whole lot has changed-so much so that many well-known and well-respected Flash designers are already calling Flash 8 the most significant Flash release in years!

Most of the new features do not involve the look and feel of the interface, but the things you can do with it.

For the designer or digital artist looking to create a web presence with Flash, there is a whole lot of new stuff. First, you now have lots of little tweaks to the tools and general interface that make it all easier to work with. Changes to gradient production, tweens (custom easing), the way vectors are drawn (Object Drawing), and tabbed panels are some examples of this.

Second, there are totally new features such as filters and other effects that will revolutionize your workflow. Stuff you would normally have had to do in a separate application, or do manually (such as adding drop shadows or bevels to buttons, or creating logos) can be done quickly and easily within Flash. Better still, doing them in Flash results in effects that are very bandwidth friendly. Further, all the new visual effects can be animated, allowing you to create animated content that was just not possible with previous versions of Flash. If you've ever used Photoshop layer blend and filter effects, you are in for a happy surprise-Flash 8 supports them, and-get this-you can animate these effects in real time! Not only can you apply these effects to bitmaps and vectors (including vector animations), but you can also apply most of them to video as well!

Third, there is now an updated scripting help system, called Script Assist. This helps nonprogrammers to write ActionScript, Flash's scripting language. ActionScript is the doorway to many of Flash's high-end visual effects, as well as the thing that allows you to add that all-important ingredient, interactivity, to your Flash content. Script Assist is one way of getting through that doorway with the minimum of fuss (and more importantly, without a lot of programming knowledge!).

One of the most significant subsets of the new enhancements for designers are those enhancements that are within the Flash Player itself.

The Flash 8 Player has several optimizations that allow you to create animations and user interfaces that run much faster than previous Flash content. This allows you to create more complex effects, bigger and better sites, or just simply smoother, more fluid animations. The Flash 8 Player now lets you import PNG files at runtime, allowing you to import images with embedded alpha channels on the fly. The Flash 8 Player also has much improved video facilities, a better sound engine, and a better text rendering engine that results in clearer, sharper text.

Of course, because the new features in Flash 8 are not obvious, it makes having a guide to help you along all the more important. That's where this book comes in! 


\section{Our aims and philosophy in this book}

As its title suggests, the aim of this book is to give you a solid, extensible foundation in Flash design, implementation, and programming. We believe that Flash is too complex a tool to cover definitively from scratch in 1,000 pages, let alone 600-odd. We want to provide a rock-steady foundation: an indepth treatment of the core aspects of using Flash rather than an overview of each and every feature.

We believe in creating a reliable foundation so you can understand Flash more fully and absorb and internalize the material we cover. We're not going to list every menu option and cover every single Actionscript command in immense detail. We're going to concentrate on the core of learning Flash successfully, taking you from a zero knowledge of Flash to being able to put up a website you can be proud of.

Everyone knows that the best way to learn is to play and practice. It's no good if someone just tells you what to do-to master Flash, you have to use it. This book follows that philosophy by providing examples and tutorials in every chapter, and on every topic we cover. It's another well-known fact that although small examples are fun and can help you learn, it's difficult to apply those examples in the real world when you've finished the book. So, at the end of each chapter, you'll be able to apply the things you've just learned to the case study project. Each case study is an opportunity for you to put the information you learn in each chapter into building a complete and fully functional website that you can use as your online portfolio:

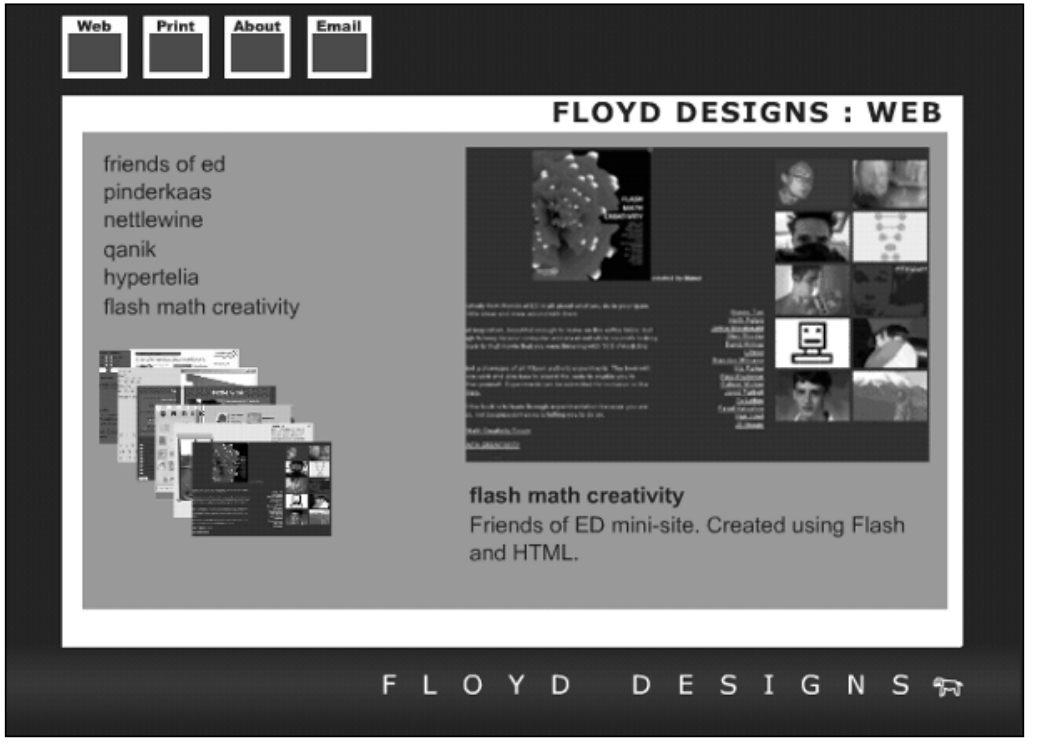

The website you'll create will have a full navigation menu and animated content, and it will dynamically load in images and text files using ActionScript. As it's created, this real-world example will reinforce the core skills you learn in this book.

We believe that by learning the Flash skills you need in context, you'll build the knowledge and mental adaptability to fit your expanding knowledge and specialization into a structured and reliable framework. 


\section{How to use this book}

To use this book, all you'll need is a copy of Macromedia Flash 8 and a computer to run it on. This book is written for either Flash Basic or Flash Professional. Most of the book can be completed using only Flash Basic, but a small subset of the features we will discuss are specific to Flash Professional. If you have Flash Basic installed, we recommend that you revisit the Flash Professional specific sections using the 30-day Flash 8 trial available at www.macromedia.com (the trial version allows you to trial either Flash Basic or Flash Professional).

If you want to publish your Flash movies onto the Internet, you'll also need a connection and some web space to publish them to. Your Internet service provider (ISP) will be able to sort this out for you if you have any problems.

The case study you'll create contains an animated introduction, interactive buttons, and examples of dynamic masking using Actionscript, and it will be fully optimized for publishing to the Web. Its modular nature means that you can easily go back and find the specific functionality that you're looking for and modify it or replace it with something completely different. If, for example, you want to use the buttons in a different website or reuse any of the animated effects, you can easily flip to the relevant chapter for a recap on how to do it, and then just pull the desired part out of the one movie and incorporate it in the other.

You don't have to download anything to use this book, but we've supplied support files containing the sounds and images that we've used to allow you to re-create the examples exactly as they are in the book. The case study files, and all support material, can be found available for download on this book's page at www. friendsofed.com. We'll point you to the relevant files in the chapters as necessary.

The case study project files are there so you can pick up the project at any stage in the book and work through it, or you can use them as backups if you've lost your files and don't want to have to re-create them all again. You may just want to check that your results are the same as ours. The files are arranged so that you have a pre-prepared project as the starting point for any chapter. For example, if you want to start from Chapter 5, you'd go into the appropriate folder and use the for chapter5. fla file, which contains all the work done on the case study from the beginning through to Chapter 4. Likewise, if you've just finished the Chapter 5 case study and want to check it against ours to make sure it looked right, you would use for chapter6. fla.

We also have some optional sound and video files that can be downloaded if you want to try your hand at the compression material we cover in Chapter 11. These (uncompressed) sound and video files are quite large, though, and could take a long time to download on some connections. 


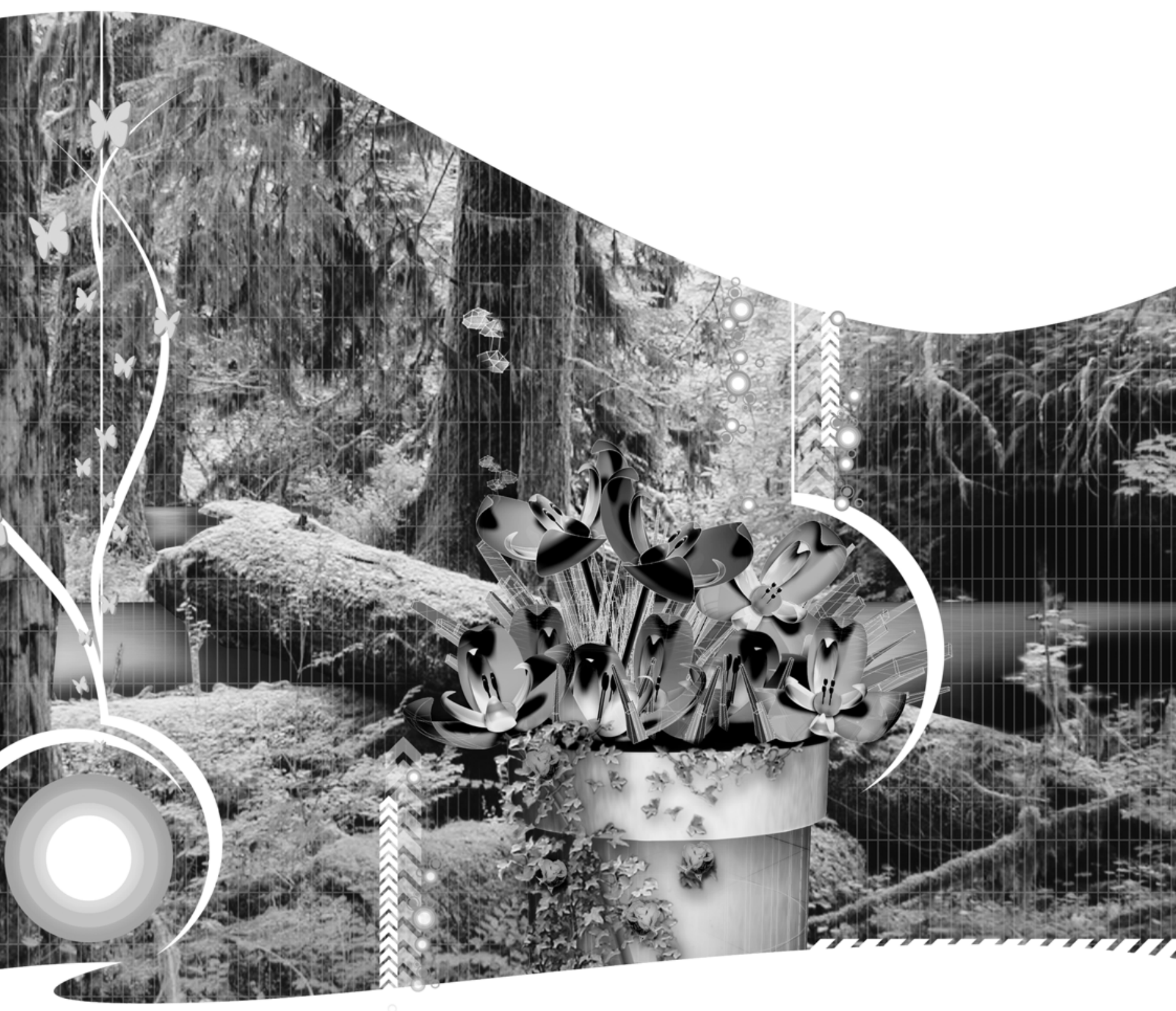

